ARTICLE

DOI: $10.1038 / \mathrm{s} 41467-017-00610-8$

\title{
Perturbed cholesterol and vesicular trafficking associated with dengue blocking in Wolbachia- infected Aedes aegypti cells
}

Vincent Geoghegan ${ }^{1,2}$, Kirsty Stainton ${ }^{2,5}$, Stephanie M. Rainey ${ }^{1}$, Thomas H. Ant ${ }^{1,2}$, Adam A. Dowle ${ }^{3}$, Tony Larson ${ }^{3}$, Svenja Hester ${ }^{4}$, Philip D. Charles ${ }^{4}$, Benjamin Thomas ${ }^{4}$ \& Steven P. Sinkins (D) ${ }^{1,2}$

Wolbachia are intracellular maternally inherited bacteria that can spread through insect populations and block virus transmission by mosquitoes, providing an important approach to dengue control. To better understand the mechanisms of virus inhibition, we here perform proteomic quantification of the effects of Wolbachia in Aedes aegypti mosquito cells and midgut. Perturbations are observed in vesicular trafficking, lipid metabolism and in the endoplasmic reticulum that could impact viral entry and replication. Wolbachia-infected cells display a differential cholesterol profile, including elevated levels of esterified cholesterol, that is consistent with perturbed intracellular cholesterol trafficking. Cyclodextrins have been shown to reverse lipid accumulation defects in cells with disrupted cholesterol homeostasis. Treatment of Wolbachia-infected Ae. aegypti cells with 2-hydroxypropyl- $\beta$-cyclodextrin restores dengue replication in Wolbachia-carrying cells, suggesting dengue is inhibited in Wolbachia-infected cells by localised cholesterol accumulation. These results demonstrate parallels between the cellular Wolbachia viral inhibition phenotype and lipid storage genetic disorders.

\footnotetext{
${ }^{1}$ MRC-University of Glasgow Centre for Virus Research, University of Glasgow, Glasgow G61 1QH, UK. ${ }^{2}$ Biomedical and Life Sciences, University of Lancaster, Lancaster LA1 4YQ, UK. ${ }^{3}$ Bioscience Technology Facility, Department of Biology, University of York, York YO10 5DD, UK. ${ }^{4}$ Sir William Dunn School of Pathology, University of Oxford, Oxford OX1 3RE, UK. ${ }^{5}$ Present address: Fera Science Ltd, Sand Hutton, York YO41 1LZ, UK. Correspondence and requests for materials should be addressed to S.P.S. (email: Steven.Sinkins@glasgow.ac.uk)
} 
W olbachia are intracellular endosymbiotic bacteria of invertebrates that are maternally transmitted. They can manipulate host reproduction to allow rapid population spread, especially using cytoplasmic incompatibility, a crossing sterility that can provide a reproductive advantage to females carrying the bacteria ${ }^{1}$. In certain host-strain combinations where high bacterial densities are reached, Wolbachia can significantly reduce the transmission of some of the most important mosquito-borne pathogens of humans including dengue virus (DENV) and chikungunya virus ${ }^{2-13}$. The $w \mathrm{Mel}$ strain has been taken to very high population frequency in wild populations of Aedes aegypti in field trials ${ }^{14}$, and is now being deployed on a larger scale in a number of countries for dengue control. It is important to gain a full understanding of the mechanisms by which Wolbachia inhibit arbovirus transmission, in order to be able to maximise the effectiveness and longevity of its use in dengue control-in particular, to be able to rapidly understand, and react effectively to, any operational failures that may arise over time.

Possible contributory factors to the phenotype include immune activation and elevated reactive oxygen species as observed in
Ae. aegypti ${ }^{4,15}$, but virus inhibition can apparently occur without observable immune activation in Ae. albopictus ${ }^{16}$ and Drosophila $^{17}$. Wolbachia likely incorporate cholesterol into their membranes as a substitute for lipopolysaccharide ${ }^{18,19}$, and it has been hypothesised based on Drosophila data that direct competition between the bacterium and viruses for this resource may be responsible for the dengue transmission-blocking phenotype ${ }^{20}$, although no supporting evidence in mosquitoes has to date been presented.

To characterise the effects of a high-density virus-blocking Wolbachia infection in Ae. aegypti and detect changes in host cellular pathways that may play a role in limiting viral replication, we performed a quantitative proteomics analysis of $w$ MelPop versus uninfected Aag2 cells. We detected perturbations in vesicular trafficking, the endoplasmic reticulum (ER) and lipid metabolism associated with Wolbachia infection. Differential expression of key proteins involved in cholesterol homeostasis indicated that Wolbachia-infected cells experience localised cholesterol accumulation and a defect in intracellular cholesterol transport. Treating Wolbachia-infected cells with 2hydroxypropyl- $\beta$-cyclodextrin, used to restore cholesterol

Table 1 Presence of Wolbachia causes differential expression of host proteins with roles in the unfolded protein response, vesicular trafficking, lipid metabolism and autophagy

Fold change wMelPop/-ve

\section{ER/unfolded protein response}

UDP-glycosyltransferase family

UDP-glycosyltransferase family

Subunit of the $\mathrm{N}$-oligosaccharyl transferase complex

Thioredoxin/protein disulfide isomerase

Poly polymerase 16

Putative udp-glucuronosyl and udp-glucosyl transferase

Oligosaccharyl transferase

Truncated ER mannose-binding lectin

Putative translocon-associated complex trap gamma subunit $\quad 1.37$

Putative calnexin 99a

Anoctamin

\section{Vesicular trafficking}

Putative lysosomal protein ncu-g1

Putative snare protein pep12/vam3/syntaxin 7/syntaxin 17

Wurst

Mucolipin-3

Vacuolar sorting protein

Putative rush hour

Syntenin

Rabkinesin-6

Tomosyn

n-myc downstream regulated (ndrg1)

myosin $\mathrm{V}$

\section{Lipid metabolism}

Apolipoprotein D

Niemann-Pick type C-2e (NPC2)/ML33

Putative lipid exporter abca1 (AAEL012701)

Putative lipid exporter abca1 (AAEL015146)

Saposin

Putative peroxisomal phytanoyl-coa hydroxylase

Putative fatty acid desaturase

Putative microsomal triglyceride transfer protein

Putative mitochondrial fatty acid anion carrier

Putative lipid particle

Fatty acid synthase

Protein phosphatase 5

Acetyl-coa carboxylase

Allergen, putative

Low-density lipoprotein receptor (Idl)

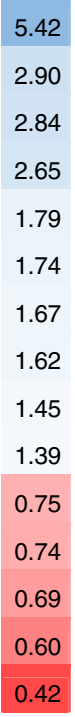

\section{Autophagy}

Vacuolar ATP synthase subunit c

Putative cysteine protease required for autophagy (ATG4B)

Tuberous sclerosis complex 2 (TSC2) 


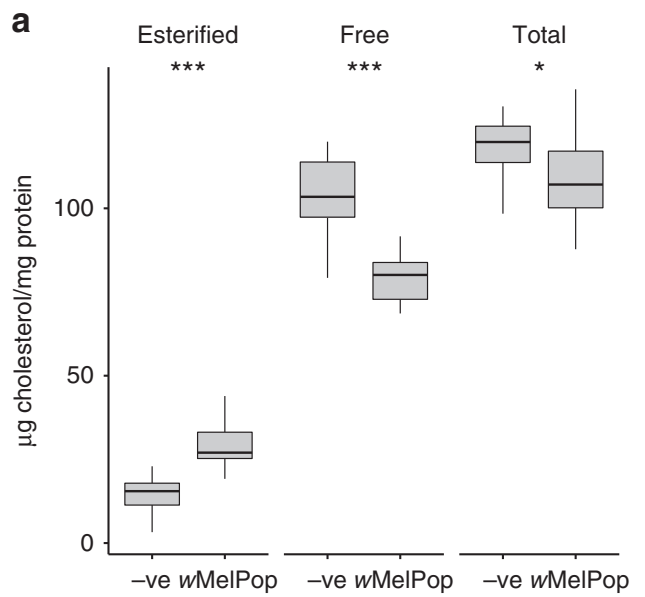

b
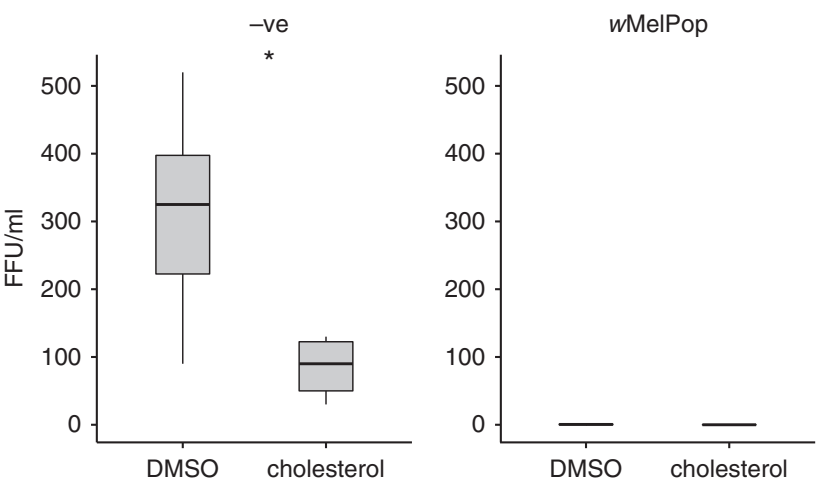

Fig. 1 Infection with Wolbachia is associated with elevated esterified cholesterol but lower free cholesterol. a Quantification of esterified, free and total cholesterol in uninfected (-ve) and wMelPop-infected Aag2 cells. Quantification was performed on 12 biological replicates of each cell line. b DENV replication in -ve or wMelPop-infected Aag2 cells. Cells were pretreated with DMSO or $50 \mu \mathrm{g} / \mathrm{ml}$ cholesterol for $48 \mathrm{~h}$ prior to infection with DENV at a multiplicity of infection of 0.1. Cell culture supernatant was harvested 5 days post infection and DENV viral titre (fluorescent focus units $/ \mathrm{ml}$ ) measured by fluorescent focus assay. Statistical significance was determined with Student's $t$-test, $n=5$

homeostasis in Niemann-Pick type C cells, rescued dengue virus replication. This suggested some similarities between Wolbachia infection and human lipid storage disorders such as Niemann-Pick type C.

\section{Results}

Proteomic analysis of Wolbachia-infected cells. To characterise the effect of Wolbachia infection on the host, we carried out a quantitative proteomic analysis of uninfected and wMelPopinfected Ae. aegypti cells. In the $w$ MelPop-carrying cell line, differential expression of 265 host proteins was observed compared to Wolbachia-free cells (Table 1, Supplementary Fig. 1, Supplementary Tables 1 and 2 and Supplementary Datas 1,2). Perturbations in vesicular trafficking, the ER as well as alterations in lipid/cholesterol metabolism were particularly notable in Wolbachia-infected cells (Table 1). Differential expression of several host proteins involved in lipid metabolism was detected including upregulation of fatty acid desaturase, which would compensate for the uptake of unsaturated fatty acids by Wolbachia, and proteins involved in catabolisis of fatty acids (saposin, phytanoylCoA hydroxylase). Conversely, fatty acid synthase was downregulated; in the presence of DENV, fatty acid synthase is upregulated and required for efficient replication ${ }^{21}$, and hence this change is likely to be antagonistic to DENV replication (Supplementary Table 2).

A lysosomal protein (ncu-g1) and syntaxin-17 (involved in autophagosome-lysosome fusion) and regulators of endocytosis (wurst and rush hour) were all upregulated in the presence of Wolbachia, while two proteins involved in exocytosis and ER to Golgi transport (rabkinesin-6 and tomosyn) were downregulated (Supplementary Table 2). Further evidence for perturbations in vesicular trafficking were observed as alterations in the levels of mucolipin-3, vacuolar sorting protein, syntenin, ndrg1 and myosin V (Fig. 1). Decreased levels of the V-ATPase regulatory subunit $\mathrm{c}$ in Wolbachia-infected cells could affect clathrinmediated endocytosis and endosomal acidification, which are critical for DENV entry ${ }^{22}$. Autophagy, the homeostatic process of encapsulation of cytoplasmic components in double-membrane vesicles for degradation, appears to be suppressed in Wolbachiainfected cells since ATG4B and TSC2 are downregulated. Autophagy is activated and required by DENV for triglyceride release from storage in cytoplasmic lipid droplets to provide energy for replication via $\beta$-oxidation ${ }^{21}$, a subversion of its usual anti-pathogen role. In contrast, Wolbachia density can be reduced by autophagy activation ${ }^{23}$, providing a selective pressure on the bacterium to suppress this pathway, with a probable antiviral side effect. Two lysosomal proteins, ncu-gl and saposin, were upregulated, which may be due to the inhibition of autophagy causing an accumulation of lysosomes.

ER proteins with roles in protein folding and glycosylation were upregulated in Wolbachia-infected cells (Table 1), suggesting the presence of ER stress and triggering of the unfolded protein response, a pathway aimed at increasing protein folding capacity to restore ER homeostasis ${ }^{24}$. Members of the uridine diphosphate glycosyltransferase family were upregulated, which play an important role in quality control of glycoproteins in the $\mathrm{ER}^{25}$. Components of the oligosaccharyltransferase complex were upregulated in Wolbachia-infected cells, involved in the cotranslational glycosylation of proteins as they enter the $\mathrm{ER}^{26}$. A major ER calcium-dependent chaperone, calnexin 99a, was upregulated as well as a protein disulfide isomerase, indicating a cellular attempt to increase protein folding activity.

Several proteins that are known to respond to changes in cholesterol or play a role in cholesterol metabolism were differentially regulated. Apolipoprotein D and ABCA1 (cholesterol efflux) and a homologue of cholesterol transporter NPC2 were upregulated in the presence of Wolbachia, while the low-density lipoprotein (LDL) receptor (cholesterol import) was downregulated (Supplementary Table 2). When cells sense an excess of cholesterol, the LDL receptor and fatty acid synthase are downregulated and cholesterol efflux transporters are upregulated. In human Niemann-Pick disease, cholesterol and/or sphingolipids accumulate in late endosomes/lysosomes due to mutations in the intracellular cholesterol transport proteins NPC1 or $\mathrm{NPC}^{27}$, and is accompanied by an increase in Apolipoprotein $\mathrm{D}$ expression. These cellular responses to cholesterol accumulation/overload mirror the protein expression changes observed in Wolbachia-infected cells, consistent with the host cell experiencing a defect in intracellular cholesterol transport.

Perturbed cholesterol homeostasis in Wolbachia-infected cells. Based on the proteomic results, cholesterol levels were measured and Wolbachia-infected cells were found to contain $\sim 100 \%$ more esterified cholesterol compared to uninfected cells (Fig. 1a, 

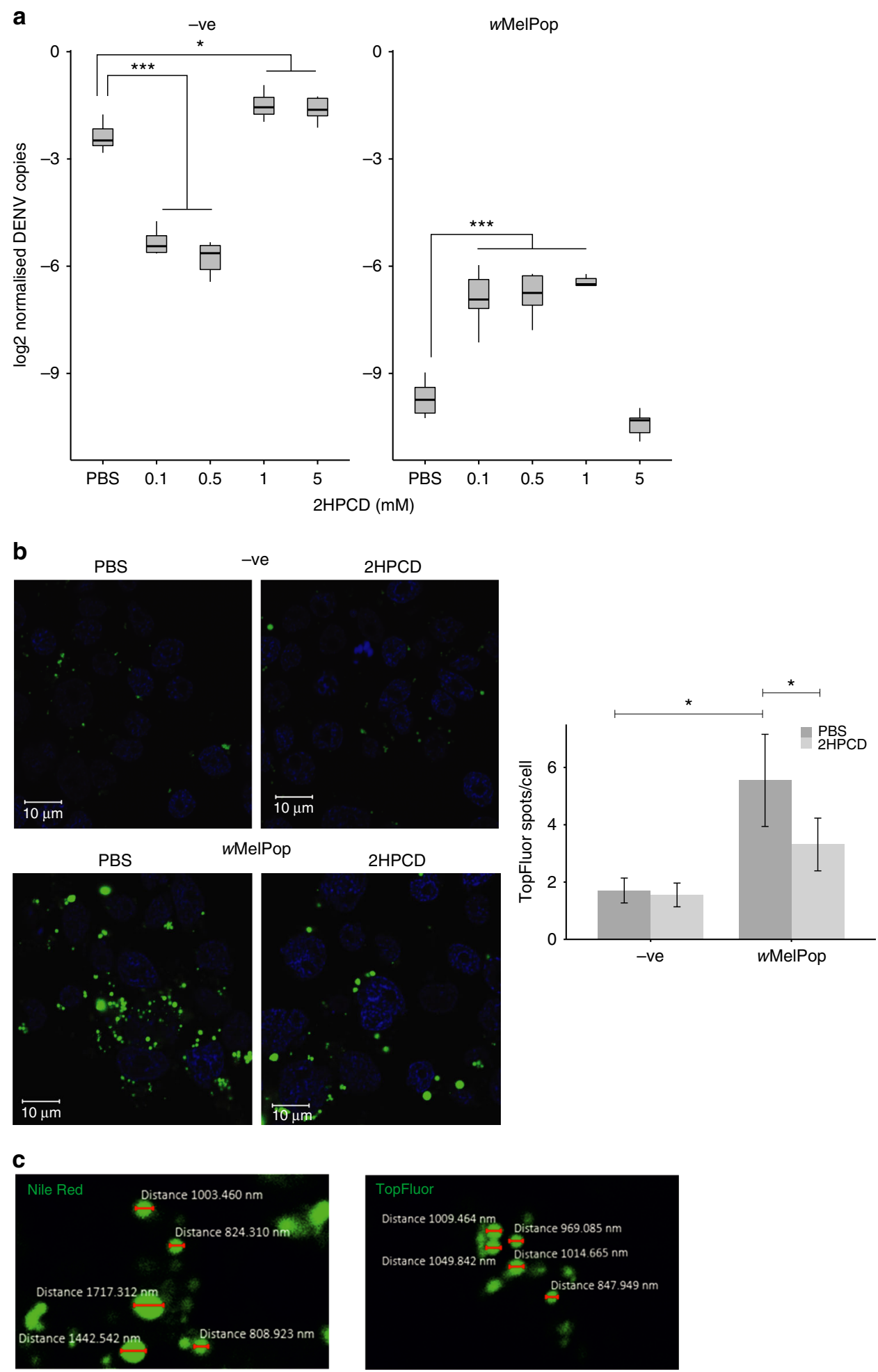

Fig. 2 Pretreatment of Wolbachia-infected Aag2 cells with low concentrations of 2-hydroxypropyl- $\beta$-cyclodextrin (2HPCD) reverses inhibition of DENV replication. a Uninfected cells (-ve) and wMelPop-infected cells were treated for $48 \mathrm{~h}$ with various concentrations of $2 \mathrm{HPCD}$ prior to addition of DENV virus at a multiplicity of infection of 0.1. Cells were harvested 5 days post infection and DENV levels measured by qPCR. Significant differences of $<0.05$ are indicated as determined by analysis of variance (ANOVA) corrected by Tukey's HSD test, $n=5$. b Cholesterol turnover imaged using TopFluor cholesterol. Uninfected and wMelPop-infected cells were pretreated with either PBS or $0.1 \mathrm{mM} 2 \mathrm{HPCD}$ for $24 \mathrm{~h}$. Cells were then labelled with TopFluor cholesterol for $30 \mathrm{~min}$ and subsequently incubated with either PBS or $0.1 \mathrm{mM} 2 \mathrm{HPCD}$ for $24 \mathrm{~h}$ before imaging by fluorescence confocal microscopy; scale bar indicates $10 \mu \mathrm{m}$. Lipid droplets were quantified as TopFluor-positive spots/cell; error bars denote s.d. Statistical significance was assessed by ANOVA corrected by Tukey's HSD test, $n=5$. c Imaging of Nile Red stained or TopFluor-treated wMelPop cells and measurement of lipid droplet size 
Table 2 Differentially expressed proteins with roles in the unfolded protein response, vesicular trafficking, lipid metabolism and autophagy in wMel-infected vs. wt Aedes aegypti midguts

Fold change $w \mathrm{Mel} /$-ve

\section{ER/unfolded protein response}

Vacuolar ATPase assembly integral membrane protein

Alpha-glucosidase

1.26

Dolichyl-diphosphooligosaccharide--protein glycosyltransferase subunit stt3b $\quad 1.21$

olichyl-diphosphooligosaccharide--protein glycosyltransferase subunit DAD1 1.21

tRNA-splicing ligase RtcB

Putative mannose lectin ergic-53

Putative calnexin 99a

Putative yip1

Reticulon-like protein

Putative proteasome non-atpase $26 \mathrm{~s}$ subunit

Putative sec23-binding domain of sec16

Heat shock protein

Peptidylprolyl isomerase

Putative transport and golgi organization protein 1

Gp210

Vesicular trafficking

Putative syntaxin 1a

Putative golgi protein

Putative neurobeachin

1.15

Vesicle protein sorting-associated

1.15

Use1

Putative vesicle coat complex copi alpha subunit

1.13

Putative nipsnap

\section{Lipid metabolism}

Niemann-Pick type C2 (AAEL015136)

Niemann-Pick type C2 (AAEL009760)

Sterol carrier protein 2-like 2

Niemann-pick C1

Palmitoyltransferase

Lipid droplet associated hydrolase

Sterol carrier protein 2-like 3 variant 1

Putative oxysterol binding protein

Phosphoacetylglucosamine mutase

Putative very long-chain acyl-coa synthetase/fatty acid transporter

Glycerol-3-phosphate acyltransferase 3-like

Putative $85 \mathrm{kDa}$ calcium-independent phospholipase a2

Putative lipid particle

Sterol carrier protein- $X$

Acyl-coenzyme A oxidase

Putative fatty acid desaturase

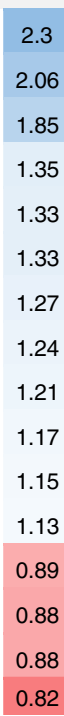

\section{Autophagy}

Putative puromycin sensitive aminopeptidease

Rabconnectin

Vacuolar H+-ATPase V1 sector subunit D

Cathepsin L
15

1.13

Fold changes in wMelPop relative to uninfected cells are coloured according to increased (blue) or decreased (red) levels

$p=0.000022$, Student's $t$-test), although free cholesterol levels were decreased by $\sim 24 \%$ in Wolbachia-infected cells ( $p=$ 0.000047 , Student's $t$-test). Total cholesterol levels were $\sim 9 \%$ lower in Wolbachia-infected cells ( $p=0.032$, Student's $t$-test). The increase in levels of esterified cholesterol in infected cells is consistent with hypercholesterolaemia, since cholesteryl esters are the primary storage and transport forms of cholesterol ${ }^{28}$. Supplementation of Aag2 wMelPop cells with cholesterol did not rescue DENV replication (Fig. 1b), while in uninfected Aag2 cells cholesterol supplementation inhibited DENV replication, as previously reported $^{29}$.

Perturbed cholesterol homeostasis can cause ER stress ${ }^{30}$, but the reverse is also true, with ER stress being associated with lipid dysregulation and an increase in cellular lipid droplet content ${ }^{31}$. It is therefore difficult to precisely dissect the hierarchy of perturbations in
Wolbachia-infected cells; however, it is likely that both ER stress and disrupted cholesterol homeostasis negatively impact DENV replication.

There is evidence that cholesterol plays an important role in entry of DENV and other flaviviruses to mammalian cells and is required in the DENV viral envelope ${ }^{29,32}$; it is also likely that they require particular lipid and cholesterol profiles for exit from the cell ${ }^{33}$. Specific cellular cholesterol profiles are required by arboviruses and both cholesterol depletion and excess can inhibit DENV - the latter probably by changing membrane rigidity ${ }^{29}$. The 2-hydroxypropyl- $\beta$-cyclodextrin (2HPCD) is a cholesterolbinding agent routinely used to modulate cellular cholesterol content $^{34}$, depending on the cyclodextrin/cholesterol molar ratio, ranging from cholesterol depletion to enrichment. At high concentrations $(>1 \mathrm{mM})$, it acts as a cholesterol sink and can 
extract cholesterol from membranes, while at lower concentrations, it can also act as a cholesterol shuttle, transporting cholesterol between membranes ${ }^{35-37}$. At lower concentrations $(<1 \mathrm{mM}), 2 \mathrm{HPCD}$ shows efficacy in the treatment of mouse models of Niemann-Pick type $\mathrm{C}$ disease ${ }^{38}$, probably by releasing trapped cholesterol into the cytosol ${ }^{33}$.

DENV rescue in Wolbachia-infected cells with 2HPCD. Our results showed upregulation of a Niemann-Pick type C-related protein and an accumulation of stored cholesterol in Wolbachiainfected cells. 2HPCD was therefore added to Ae. aegypti cells, and rescue of DENV replication in $w$ MelPop-containing cells was observed (Fig. 2a). In contrast, the same concentrations of 2HPCD strongly inhibited DENV in control Wolbachia-free Ae. aegypti cells (Fig. 2a), in agreement with a previous study ${ }^{32}$. Importantly, Wolbachia density was not significantly affected by the cyclodextrin treatment, thus ruling out a density-mediated mechanism of rescue of inhibition (Supplementary Fig. 2). $2 \mathrm{HPCD}$ also rescued DENV replication in $w$ MelPop-containing cells at the infectious particle level (Supplementary Fig. 3). To visualise cholesterol dynamics, pulse labelling of uninfected and $w$ MelPop-infected Aag2 cells with a fluorescently labelled cholesterol analogue was undertaken and the cells imaged $48 \mathrm{~h}$ later. The wMelPop cells showed significantly higher intracellular accumulation of the fluorescent cholesterol in large spots within the cell (Fig. 2b), and this accumulation was reduced upon 2HPCD treatment. The labelled cholesterol did not colocalise with the rab7 marker of late endosomes (Supplementary Fig. 4). We hypothesised that TopFluor was localising to lipid droplets due to the round shape and size of the spheres observed. In order to determine if this was the case, we stained $w$ MelPop cells with the dye Nile Red. This dye specifically binds lipids and fluoresces green/yellow when binding neutral lipids such as those stored in lipid droplets ${ }^{39}$. Comparison of the spheres observed under Nile Red and TopFluor staining (Fig. 2c) indicate that TopFluor is localising to lipid droplets. Further to this, three-dimensional (3D) reconstruction of TopFluor labelled images showed, upon 2HPCD treatment, a clear diffusion of green fluorescence observed in $w$ MelPop cells and not in negative cells, suggesting a reduction in lipid droplet accumulation of TopFluor (Supplementary Fig. 5).

Proteomic analysis of Wolbachia-infected Ae. aegypti midguts. In order to examine whether similar Wolbachia-induced changes could be observed in vivo, a quantitative proteomics analysis of uninfected and $w \mathrm{Mel}$-infected Ae. aegypti midguts was performed. The $w \mathrm{Mel}$ strain is a less efficient dengue blocker than $w$ MelPop and is maintained at a lower density, but is currently the strain most commonly being utilised for field releases ${ }^{5}, 9-13$. As midguts are the first tissue encountered by DENV following its ingestion in a bloodmeal, any Wolbachia-associated perturbations would significantly impact on DENV replication and dissemination in the host. In total, 3,562 unique proteins were quantified, of which 434 showed differential expression in $w \mathrm{Mel}$ vs. uninfected midguts (Table 2, Supplementary Table 3 and, Supplementary Data 3). Wolbachia-associated changes were observed in cholesterol homeostasis, ER proteins, vesicular trafficking and autophagy, suggesting similar perturbations were induced by Wolbachia in the midguts and cell line (Table 2).

In $w$ Mel-carrying midguts there was upregulation of several Niemann-Pick proteins and proteins involved in intracellular sterol transport, with the exception of sterol carrier protein $\mathrm{X}$, which was downregulated. As observed in the cell line, several ER proteins were upregulated including components of the oligosaccharyl transferase complex (stt3b, DAD1) and proteins responsible for restoring ER homeostasis as part of the unfolded protein response (RtcB, calnexin 99a, heat shock protein, peptidylprolylisomerase). Interestingly, several secreted proteins (trypsin 5G1, chymotrypsin-1, chymotrypsin-2, vitellogenin, Supplementary Data 3) were upregulated in the Wolbachiacarrying midguts. These proteins are normally upregulated following a bloodmeal; their upregulation in non-blood-fed Wolbachia-infected mosquitoes could be a result of a defect in protein secretion, possibly as a result of altered ER homeostasis, leading to intracellular accumulation. Elevated trypsin 5G1 prior to a bloodmeal may further reduce DENV entry and replication in the midgut ${ }^{40}$.

Several proteins whose human homologues are mutated in lysosomal storage disorders were found to be upregulated in the presence of Wolbachia, providing further evidence of perturbed lipid metabolism/vesicular trafficking (Supplementary Table 3). In particular, sphingomyelin phosphodiesterases, enzymes deficient in Niemann-Pick disease type $\mathrm{A}^{41}$, were upregulated. These data strongly suggest that the perturbations observed in the cell line also occur in vivo, and also demonstrate that they are not restricted to the $w$ MelPop infection, which reaches unusually high density and affects the transcription of an unusually large number of host genes compared to lower-density virus-blocking Wolbachia infections ${ }^{42}$.

\section{Discussion}

A number of the proteins affected by the presence of Wolbachia are mutated or deficient in human lysosomal storage disorders such as Niemann-Pick disease, suggesting some parallels between these cellular states. As is thought to be the case in 2HPCD treatment of the Niemann-Pick type $\mathrm{C}$ disorder, the cyclodextrin treatment of Wolbachia-infected cells is likely to act as a shuttle and release trapped cholesterol into the cytosol, facilitating virus replication. Our data suggest Wolbachia-mediated inhibition occurs early in the DENV replication cycle, consistent with a recent study by Rainey et al. ${ }^{43}$.

Since lipids are degraded in late endosomes, perturbations in vesicular trafficking at this stage would likely manifest as a dysregulation of several lipid classes ${ }^{44}$, and manipulation of other lipids should be further investigated in future work. Molloy et al. $^{45}$ performed lipidomic analysis of Wolbachia-infected Ae. albopictus cells revealing depletion of ceramides and altered levels of some sphingomyelin species. Data presented here suggest this may be related to defects in late endosome processing of sphingomyelin to ceramide. Wolbachia-infected cells showed an upregulation of sphingomyelin phosphodiesterases, the enzyme responsible for hydrolysing sphingomyelin to ceramide, indicating a host attempt to correct defects in late endosome lipid processing and restore cellular homeostasis. Molloy et al. ${ }^{45}$ also found that phosphatidylcholine and phosphatidylethanolamine levels were strongly perturbed. Treatment of cells with U18666A induces a Niemann-Pick type C-like phenotype, inhibits Dengue replication and similarly causes dysregulation in phosphatidylcholine and phosphatidylethanolamine due to defects in late endosome lipid trafficking ${ }^{46}$.

In contrast to the Aedes data presented here, supplementation with cholesterol reduced Wolbachia-mediated protection from the pathogenic virus DCV in Drosophila and increased viral replication ${ }^{20}$. Given that this virus is not related to the Flavivirus DENV, there are likely to be differences in the mechanisms of cell entry/replication and degree of cholesterol dependence that will influence the interactions between virus, Wolbachia and host cells. In addition, the effect of cholesterol supplementation on DCV titre in uninfected Drosophila was not investigated in the study of Caragata et al. ${ }^{20}$, making it unclear if the effect observed 
was a reversal of Wolbachia-mediated inhibition or a general enhancement of viral replication. The differences observed between Drosophila and Aedes could also be related to fluctuations in Wolbachia density following dietary cholesterol treatment in Drosophila, rather than a direct effect ${ }^{20}$.

In both the Wolbachia-infected Ae. aegypti cell line and midguts, perturbations were observed in the ER, vesicular trafficking, lipid metabolism and autophagy. The good overlap between the two data sets at the level of pathways affected but not at the level of individual differentially expressed proteins is likely due to the different proteomic composition of a cell line vs. midguts and the different lysis and quantitation methods used.

The detected ER perturbation in Wolbachia-infected cells is of interest given that DENV uses the ER for synthesis and maturation of proteins ${ }^{47}$ and data show close association of Wolbachia with the ER membranes ${ }^{48}$. Lipid droplets are ERderived protein and lipid storage organelles targeted by DENV structural protein $\mathrm{C}^{49}$; therefore, the dysregulated cholesterol trafficking and accumulation of cholesterol rich lipid droplets observed in Wolbachia-carrying cells may interfere with proper localisation of DENV protein C, impairing replication. Cholesterol-rich lipid droplets were dispersed after 2HPCD treatment and 2HPCD has been widely used to modulate cholesterol homeostasis; however, it should be noted that 2HPCD contains a hydrophobic core which may bind to lipids other than cholesterol.

Overall, the data presented demonstrate that in Ae. aegypti cells, Wolbachia infection interferes with intracellular cholesterol trafficking, leads to an accumulation of intracellular cholesterol stores, perturbs the ER and interferes with vesicular trafficking and lipid metabolism. Treatment with cyclodextrin provides a clear indication that these changes are antagonistic to DENV.

\section{Methods}

Cell culture. Ae. aegypti Aag2 cells (origin J. Peleg, Israel Institute for Biological Research, Israel, 1970s; obtained from A. Kohl, Glasgow University) were cultured at $28^{\circ} \mathrm{C}$ in Schneider's Drosophila medium (Pan Biotech Ltd) supplemented with $10 \%$ fetal bovine serum (FBS; Sigma) and 200 units $/ \mathrm{ml}$ penicillin $200 \mu \mathrm{g} / \mathrm{ml}$ streptomycin (Sigma). An Aag2 subline was transinfected with wMelPop Wolbachia from Drosophila melanogaster line $w^{1118}$, as previously described ${ }^{16}, 50$. Briefly, $w$ MelPop-infected $D$. melanogaster embryos were collected and dechorionated using a $2.1 \%$ sodium hypochlorite solution for $2 \mathrm{~min}$. Embryos were rinsed three times with distilled water, immersed in $70 \%$ ethanol for $15 \mathrm{~s}$ and washed three times with phosphate-buffered saline (PBS). Embryos were homogenised in a mini Dounce homogeniser (Wheaton) using a tight pestle for 2-3 min. Embryo homogenate was overlaid on $75 \%$ confluent Aag2 cells in 12-well plates. Plates were centrifuged at $2,000 \times g$ for $1 \mathrm{~h}$ at room temperature (RT) and cells subsequently incubated for $24 \mathrm{~h}$ at $28^{\circ} \mathrm{C}$. Absolute Wolbachia density in the Aag2 wMelPop line was $106 \pm 30$ (s.d., $n=3$ ) Wolbachia surface protein/homothorax (wsp/HTH) as measured by quantitative PCR (qPCR; see qPCR methods). Vero cells (ECACC catalogue no. 85020205) were cultured at $37^{\circ} \mathrm{C}$ with $5 \% \mathrm{CO}_{2}$ in Dulbecco's modified Eagle's medium (DMEM) (Sigma) with $2 \mathrm{mM}$ L-glutamine and $10 \%$ FBS in a humidified incubator.

Experimental design and statistical rational. A total of four independent biological replicates (flasks) were processed, sufficient to estimate differential fold changes using the LIMMA software package ${ }^{51}$. Label swapping was performed for each biological replicate to eliminate systematic label quantification bias. Five independent biological replicates of uninfected and wMel-infected Ae. aegypti midguts were tandem mass tag (TMT) labelled (Thermo) and processed for mass spectrometry, sufficient to estimate statistically significant fold changes using ScaffoldQ+ 4.0 (Proteome Software Inc.).

Cell lysis, digestion and peptide labelling. Cells were harvested by scraping at $50 \%$ confluency $\left(15 \times 10^{6}\right.$ per replicate, 4 replicates per cell line $)$, washed twice in PBS and stored at $-80^{\circ} \mathrm{C}$. Cell pellets were thawed on ice and lysed in $300 \mu \mathrm{l} 0.2 \%$ PPS silent surfactant (Expedeon) in $50 \mathrm{mM}$ triethylammonium bicarbonate

(TEAB) and vortexed. Lysates were heated at $95{ }^{\circ} \mathrm{C}$ for $5 \mathrm{~min}$, cooled on ice and sonicated with a microtip sonicator 3 times for $10 \mathrm{~s}$ each. Protein was measured using the bicinchoninic acid (BCA) assay (Pierce). Samples were reduced with 5 $\mathrm{mM}$ dithiothreitol (DTT) for $30 \mathrm{~min}$ at $50^{\circ} \mathrm{C}$ and alkylated with $15 \mathrm{mM}$

2-Chloracetamide for $30 \mathrm{~min}$ in the dark. Proteins were digested overnight at $37^{\circ} \mathrm{C}$ with trypsin (Sigma)/protein of 1:75 in the presence of $1 \mathrm{mM} \mathrm{CaCl} \mathrm{m}_{2}$. PPS surfactant was cleaved with $0.5 \%$ trifluoroacetic acid (TFA) for $1 \mathrm{~h}$ at RT. Digests were centrifuged at $18,000 \times g$ for $15 \mathrm{~min}$ and the supernatant dried. Dried peptides were resuspended in $1 \mathrm{ml} \mathrm{5 \%}$ formic acid per quantitation channel. Peptides were stable isotope dimethyl labelled according to Boersema et al. ${ }^{52}$. Briefly, labelling was carried out in $200 \mathrm{mg} \mathrm{C}_{18}$ columns. Columns were first conditioned with acetonitrile followed by buffer A ( $0.6 \%$ (v:v) acetic acid). Peptides were loaded onto columns and washed with buffer A before being labelled with $5 \times 1 \mathrm{ml}$ of either $4 \%$ (v:v) $\mathrm{CH}_{2} \mathrm{O}, 0.6 \mathrm{M} \mathrm{NaBH}_{3} \mathrm{CN}$ (light), $4 \%$ (v:v) $\mathrm{CD}_{2} \mathrm{O}, 0.6 \mathrm{M} \mathrm{NaBH}{ }_{3} \mathrm{CN}$ (medium) or $4 \%(\mathrm{v}: \mathrm{v})^{13} \mathrm{CD}_{2} \mathrm{O}, 0.6 \mathrm{M} \mathrm{NaBD}{ }_{3} \mathrm{CN}$ (heavy) in $50 \mathrm{mM}$ sodium phosphate $\mathrm{pH} 7.4$ Columns were washed with buffer A and peptides eluted with $50 \%$ (v:v) acetonitrile $0.6 \%$ (v:v) acetic acid. Samples were mixed according to a 1:1 protein ratio and peptides dried.

Midgut lysis, digestion and peptide labelling. Midguts were dissected from 10 day-old non-blood-fed female Ae. aegypti and immediately frozen at $-80{ }^{\circ} \mathrm{C}$. For each line (uninfected or $w \mathrm{Mel}$ infected), midguts from 20 mosquitoes were used for a biological replicate. Midguts were lysed by sonicating $3 \times 15 \mathrm{~s}$ in $8 \mathrm{M}$ urea and 50 $\mathrm{mM}$ TEAB. Protein was measured by BCA assay (Pierce). Samples were reduced with $5 \mathrm{mM}$ DTT for $30 \mathrm{~min}$ at $50{ }^{\circ} \mathrm{C}$ and alkylated with $15 \mathrm{mM}$ Iodoacetamide for $30 \mathrm{~min}$ in the dark. Urea was diluted to $1.5 \mathrm{M}$ with $50 \mathrm{mM}$ TEAB and trypsin/Lys $\mathrm{C}$ (Promega) added at an enzyme/protein ratio of 1:25. Proteins were digested overnight at $37^{\circ} \mathrm{C}$. Digests were acidified with addition of TFA to $0.5 \%$ (v:v) and clarified by centrifugation for $7 \mathrm{~min}$ at $18,000 \times g$. Peptides were desalted using Strata $50 \mathrm{mg} \mathrm{C}_{18}$ cartridges (Phenomenex). Cartridges were prepared by passing through $3 \mathrm{ml}$ acetonitrile, $2 \mathrm{ml}$ aqueous $80 \%$ (v:v) acetonitrile $0.1 \%$ (v:v) TFA and $2 \mathrm{ml}$ aqueous $0.1 \%$ (v:v) TFA. Peptides were loaded and cartridges washed with $2 \times$ $0.25 \mathrm{ml}$ aqueous $0.1 \%$ TFA. Peptides were eluted with aqueous $80 \%$ (v:v) acetonitrile $0.1 \%$ (v:v) TFA and dried. Peptides were resuspended in $50 \mathrm{mM}$ TEAB at a concentration of $43 \mu \mathrm{g} / 100 \mu \mathrm{l}$. A $100 \mu \mathrm{l}$ aliquot of peptide solution from each sample was labelled using TMT reagents (TMT-10plex, Thermo Fisher). For each sample, $0.8 \mathrm{mg}$ of TMT reagent was resuspended in $41 \mu \mathrm{l}$ acetonitrile and added to peptides, and labelling took place over $1 \mathrm{~h}$ at RT. Excess labelling reagent was quenched with $8 \mu \mathrm{l}$ aqueous $5 \%$ (v:v) hydroxylamine for $15 \mathrm{~min}$ at RT. Labelled peptides were dried prior to high $\mathrm{pH}$ reversed-phase fractionation.

Peptide fractionation. Labelled peptides from cell line digests were fractionated by OFFGEL peptide fractionation, carried out according to the manufacturer's instructions. Briefly, peptides were resuspended in $720 \mu \mathrm{l}$ water per replicate and mixed with OFFGEL solution containing $\mathrm{pH} 3-11$ ampholytes. Then, $3 \mathrm{mg}$ of peptide was fractionated into 24 wells over a $24 \mathrm{~cm} \mathrm{pH} \mathrm{3-11} \mathrm{immobilised} \mathrm{pH}$ gradient strip. Fractions were acidified with $0.1 \%$ formic acid and desalted with StageTips.

Labelled peptides from midgut digests were fractionated using high $\mathrm{pH}$ reversed-phase spin columns according to the manufacturer's instructions (Pierce) Column was washed twice with $300 \mu \mathrm{l}$ acetonitrile and twice with aqueous $0.1 \%$ (v:v) TFA. Peptides were resuspended in $300 \mu \mathrm{l}$ aqueous $0.1 \%$ (v:v) TFA and applied to the column by centrifugation for $2 \mathrm{~min}$ at $3,000 \times \mathrm{g}$. Peptides were washed with $300 \mu \mathrm{l}$ water, then $300 \mu \mathrm{l} 5 \%$ acetonitrile $0.1 \%$ triethylamine and eluted with $300 \mu \mathrm{l}$ each of aqueous (v:v) 10, 12.5, 15, 17.5, 20, 22.5, 25, 50\% acetonitrile $0.1 \%$ triethylamine. Fractions were dried down for analysis by mass spectrometry.

Mass spectrometry. Peptides from cell line samples were resuspended in $0.1 \%$ formic acid and resolved on a $\mathrm{C}_{18}$ reverse-phase PepMap RSLC $50 \mathrm{~cm} \times 75 \mu \mathrm{m} \times 2$ $\mu \mathrm{m}, 100 \AA$ A Easy-Column (Thermo Scientific) using a linear gradient of 5-55\% Buffer B (aqueous $80 \%$ (v:v) acetonitrile, $0.1 \%$ (v:v) formic acid) at $300 \mathrm{nl} / \mathrm{min}$ over 130 min run on an Ultimate 3000 RSLC nano (Dionex) system coupled to QExactive mass spectrometer (Thermo Scientific). The mass spectrometer was operated in a 'Top 10' data-dependent acquisition mode with dynamic exclusion enabled $(40 \mathrm{~s})$. Survey scans (mass range $\mathrm{m} / \mathrm{z} 300-1,650$ ) were acquired at a resolution of 70,000 at 200 Th with the 10 most abundant multiply charged $(+2$ and +3$)$ ions selected with a $3 \mathrm{Th}$ isolation window for high-energy collisional dissociation (HCD) fragmentation. Tandem mass spectrometry (MS/MS) scans were acquired at a resolution of 17,500 at $200 \mathrm{Th}$.

Peptides from midgut samples were resuspended in $0.1 \%$ formic acid and loaded onto an UltiMate 3000 RSLCnano HPLC system (Thermo) equipped with a PepMap $100 \AA_{\mathrm{C}_{18}}, 5 \mu \mathrm{m}$ trap column $(300 \mu \mathrm{m} \times 5 \mathrm{~mm}$, Thermo $)$ and a PepMap, 2 $\mu \mathrm{m}, 100 \AA \mathrm{C}_{18}$ Easy Nanonanocapillary column $(75 \mu \mathrm{m} \times 150 \mathrm{~mm}$, Thermo). The trap wash solvent was $0.05 \%(\mathrm{v}: \mathrm{v})$ aqueous TFA and the trapping flow rate was 15 $\mu \mathrm{l} / \mathrm{min}$. The trap was washed for 3 min before switching flow to the capillary column. Separation used gradient elution of two solvents: solvent A, aqueous $1 \%$ (v:v) formic acid; solvent B, aqueous $80 \%$ (v:v) acetonitrile containing $1 \%$ (v:v) formic acid. The flow rate for the capillary column was $300 \mathrm{nl} / \mathrm{min}$ and the column temperature was $40{ }^{\circ} \mathrm{C}$. The linear multi-step gradient profile was: $3-10 \%$ B over 8 min, $10-35 \%$ B over $125 \mathrm{~min}, 35-65 \%$ B over $50 \mathrm{~min}, 65-99 \%$ B over $7 \mathrm{~min}$ and then proceeded to wash with $99 \%$ solvent B for $4 \mathrm{~min}$. The column was returned to initial conditions and re-equilibrated for $15 \mathrm{~min}$ before subsequent injections.

The nanoLC system was interfaced with an Orbitrap Fusion hybrid mass spectrometer (Thermo) with an EasyNano ionisation source (Thermo). Positive 
electrospray ionisation (ESI)-MS, $\mathrm{MS}^{2}$ and $\mathrm{MS}^{3}$ spectra were acquired using Xcalibur software (version 4.0, Thermo). Instrument source settings were: ion spray voltage, $1,900 \mathrm{~V}$; sweep gas, $0 \mathrm{Arb}$; ion transfer tube temperature, $275^{\circ} \mathrm{C} . \mathrm{MS}^{1}$ spectra were acquired in the Orbitrap with: 120,000 resolution, scan range: $\mathrm{m} / \mathrm{z}$ $380-1,500$; automatic gain control (AGC) target, $2 \mathrm{e}^{5}$; max fill time, $50 \mathrm{~ms}$. Datadependant acquisition was performed in top speed mode using a $4 \mathrm{~s}$ cycle, selecting the most intense precursors with charge states $>1$. Dynamic exclusion was performed for $50 \mathrm{~s}$ post-precursor selection and a minimum threshold for fragmentation was set at $3 \mathrm{e}^{4}$. $\mathrm{MS}^{2}$ spectra were acquired in the linear ion trap with: scan rate, turbo; quadrupole isolation, $1.2 \mathrm{~m} / z$; activation type, collision-induced dissociation; activation energy: $35 \%$; AGC target, $1 \mathrm{e}^{4}$; first mass, $120 \mathrm{~m} / z$; max fill time, $50 \mathrm{~ms} . \mathrm{MS}^{3}$ spectra were acquired in multi notch synchronous precursor mode $\left(\mathrm{SPS}^{3}\right.$ ), selecting the 5 most intense $\mathrm{MS}^{2}$ fragment ions between 400 and $1,000 \mathrm{~m} / z$. SPS $^{3}$ spectra were measured in the Orbitrap mass analyser using: 50,000 resolution, quadrupole isolation, $2 \mathrm{~m} / z$; activation type, $\mathrm{HCD}$; collision energy, 65\%; scan range: $m / z$ 110-500; AGC target, $5 \mathrm{e}^{4}$; max fill time, $86 \mathrm{~ms}$. Acquisitions were arranged by Xcalibur to inject ions for all available parallelisable time.

MS data analysis. MS data files from cell line samples were de-isotoped and charge deconvoluted with Proteome Discoverer 1.4 (Thermo). Files were searched using Mascot 2.4.1 against a concatenated and reversed decoy Ae. aegypti and Wolbachia wMel database containing 18,082 sequences. Cysteine carbamidomethylation and protein $\mathrm{N}$-terminal acetylation were set as fixed modifications. Oxidation (M), dimethyl (K, N-termini), dimethyl-4 (K, N-termini) and dimethyl-8 (K, N-termini) were set as variable modifications. Precursor mass tolerance was 20 p.p.m., and fragment mass tolerance was $0.1 \mathrm{Da}$. Two mis-cleavages were allowed. Peptide cutoff score was 10 and protein relevance threshold was 20, false discovery rate (FDR) was $1.23 \%$. The structure of Proteome Discoverer.msf files was viewed with DbVisualizer (DbVis Software AB). The 'Pre-

cursorIonQuanResults' table was exported. For each peptide with a quantitation status of 'used', the 'QuanResultID' was used to obtain light, medium and heavy peak areas, summed within each 'QuanChannelID'. For each biological replicate, proteins with $>=3$ 'QuanResultID' associated were retained. Peptide intensities for each protein were summed within quantitation channels. Differentially expressed proteins were determined using LIMMA ${ }^{51}$, with normalizeWithinArrays (method=loess) and normalizeBetweenArrays (method=quantile). $P$-values were calculated for protein fold changes, adjusted for multiple hypothesis testing with the Benjamini-Hochberg method ${ }^{53}$, and fold changes with an adjusted $P$-value $<0.05$ were accepted. From the cell line, 3,785 proteins, representing $~ 28 \%$ of the proteome, were identified and quantified in at least 3 biological replicates.

For midgut-derived TMT data peak lists were converted from centroided raw to .mgf format using Mascot Distiller (version 2.6.1, Matrix Science) and $\mathrm{MS}^{3}$ spectra were concatenated into their parent $\mathrm{MS}^{2}$ spectra for database searching. Mascot Daemon (version 2.5.1, Matrix Science) was used to combine .mgf files and search against a subset of the UniProt database containing Ae. aegypti and Wolbachia w Mel proteins (17,811 sequences) using a locally running copy of the Mascot program (Matrix Science Ltd, version 2.5.1). Search criteria specified: Enzyme, trypsin; Fixed modifications, Carbamidomethyl (C), TMT6plex (N-term, $\mathrm{K})$; Variable modifications, Oxidation (M); Peptide tolerance, 5 p.p.m.; MS/MS tolerance, $0.5 \mathrm{Da}$; Instrument, ESI-TRAP. The Mascot dat result file was imported into Scaffold Q + (version 4.7.5, Proteome Software) and a second search run against the same database using X!Tandem was run. Protein identifications were filtered to require a maximum protein and peptide FDR of $1 \%$ with a minimum of two unique peptide identifications per protein. Protein probabilities were assigned by the Protein Prophet algorithm ${ }^{54}$. Proteins that contained similar peptides and could not be differentiated based on MS/MS analysis alone were grouped to satisfy the principles of parsimony. Proteins sharing significant peptide evidence were grouped into clusters. Quantification of relative protein abundance was calculated from TMT reporter ion intensities using Scaffold Q +. TMT isotope correction factors were taken from the document supplied with the reagents by the manufacturer. Normalisation was performed iteratively (across samples and spectra) on intensities, as described in ref. ${ }^{55}$. Medians were used for averaging. Spectra data were log-transformed, pruned of those matched to multiple proteins and weighted by an adaptive intensity weighting algorithm. Differentially expressed proteins were determined by applying permutation test with unadjusted significance level $p<0.05$ corrected by Benjamini-Hochberg for FDR estimation. The mass spectrometry proteomics data have been deposited to the ProteomeXchange Consortium ${ }^{56}$ via the PRIDE partner repository with the data set identifier PXD003429 (Aag2 cell data) or PXD006239 (midgut data).

Cholesterol quantification. Aag2 cells stably infected with $w$ MelPop and a rifampicin cured negative line were grown to confluency in six-well plates. Cells were washed twice with PBS prior to harvesting by scraping. In all, 12 wells of each cell type were harvested for cholesterol measurements. Then, $200 \mu \mathrm{l}$ of hexane/ isopropanol 3:2 was added to the cell pellet from half a well to extract cholesterol. Extracts were centrifuged at $10,000 \times g$ for $5 \mathrm{~min}$ at RT, the supernatant was dried in a vacuum centrifuge overnight and extracts resuspended in $100 \mu$ reaction buffer $(0.1 \mathrm{M}$ potassium phosphate $\mathrm{pH} 7.4,0.05 \mathrm{M} \mathrm{NaCl}, 5 \mathrm{mM}$ cholic acid, $0.1 \%$ Triton $\mathrm{X}-100$, Thermo Fisher). Free cholesterol was quantified using the Amplex Red Cholesterol Assay Kit (Thermo Fisher), and esterified cholesterol was quantified by including cholesterol esterase in the reactions. Cholesterol quantities were normalised to total input protein. Input protein was measured by resuspending the protein pellet left after hexane/isopropanol cholesterol extraction in $100 \mu \mathrm{l}$ RIPA buffer (Sigma). Protein content was measured using the BCA assay (Thermo Scientific).

Imaging. Uninfected and infected $w$ MelPop Aag2 cells were seeded at $3 \times 10^{5}$ cells/ well in $1 \mathrm{ml}$ FBS and penicillin/streptomycin supplemented Schneider's Drosophila medium in a 24-well poly-L-lysine-coated plate and allowed to adhere overnight. Cholesterol was labelled according to Sankaranarayanan et al. ${ }^{57}$; briefly, labelling media was prepared by adding $3.47 \mathrm{ml}$ of $20 \mathrm{mM}$ methyl-beta-cyclodextrin (Sigma) in unsupplemented Schneider's Drosophila medium to $1 \mathrm{mg}$ of 23-(dipyrrometheneborondifluoride)-24-norcholesterol (TopFluor cholesterol, Avanti Polar Lipids) to achieve a 1:40 cholesterol/cyclodextrin molar ratio. This mixture was sonicated for $6 \times 5 \mathrm{~s}$ pulses. The sample was then centrifuged at $19,000 \times g$ for 10 min to remove undissolved cholesterol. TopFluor cholesterol was then diluted 1:1 in unsupplemented Schneider's Drosophila medium. This was then added to the cells and incubated for $30 \mathrm{~min}$ to allow labelling to occur. Labelling media were then removed and either PBS or $0.1 \mathrm{mM}$ of $2 \mathrm{HPCD}$ was added. After $48 \mathrm{~h}$, the cells were washed with PBS before Vecta shield mounting media with 4',6-diamidino-2phenylindole (DAPI; Vectorlabs) was added. Images were then acquired using a Zeiss LSM 880 confocal microscope (Zeiss) with a $63 \times$ objective. Cholesterol was imaged using a $488 \mathrm{~nm}$ laser, with GaAsP detectors. Nuclei stained with DAPI were imaged using a $405 \mathrm{~nm}$ laser with GaAsP detector. All settings were obtained by first imaging uninfected Aag2 cells incubated in PBS as a standard control ensuring all data were correlated. $Z$-stack images were collected and analysed using Zen2 software (Zeiss) to create a 3D reconstruction of imaged cells after deconvolution was carried out.

Quantification was carried out by imaging 5 independent $\times 64$ images from 5 independent wells on a 24 -well optical plate. Images were analysed using Cell Profiler. A global image threshold was set using the Otsu method and images were analysed in order to identify the number of nuclei and the number of green spots corresponding to TopFluor staining. Data are presented as the number of spots per cell.

For Rab7 staining, Aag2 $w$ MelPop cells were fixed with $2.5 \%$ paraformaldehyde and permeabilised in PBS containing $0.01 \%$ Triton-X for 15 min. Cells were blocked for $1 \mathrm{~h}$ with 5\% serum before incubation with Rab7 antibody (Abcam, ab50533, 1:500). Images were obtained as stated above.

For Nile Red staining, cells were fixed and permeabilised as above. Cells were then incubated for $10 \mathrm{~min}$ in $10 \mu \mathrm{g} / \mathrm{ml}$ of Nile Red. Nile Red is known to fluoresce in green/yellow where lipid droplets are present ${ }^{39}$. Images were then acquired using a Zeiss LSM 880 confocal microscope (Zeiss) with a $63 \times$ objective as above with the 514 laser. Images were processed in Zen blue and lipid droplets measured.

DENV-2 propagation, infection and quantification. DENV-2 New Guinea C strain (Public Health England, 0006041v), propagated in Vero cells, was diluted 1:100, one round of propagation was carried out in C6/36 cells (ECACC catalogue no. 89051705) for 5 days and the resulting virus stock was centrifuged for $7 \mathrm{~min}$ at $400 \times g$ and quantified by fluorescent focus assay (FFA) ${ }^{58}$. For the FFA, Vero cells were plated at a density of $0.9 \times 10^{5}$ cells/well in 96-well special optics plates (Corning). The following day, 10-fold serial dilutions of DENV containing supernatant were prepared using DMEM with 2\% FBS. Medium was removed from Vero cells and $50 \mu \mathrm{l}$ of each serial dilution was added to wells. Plate was placed on an orbital shaker at 20 r.p.m. and left at $37^{\circ} \mathrm{C}$ for DENV to bind for $45 \mathrm{~min}$. DENV was removed and $150 \mu \mathrm{l}$ of overlay medium/well (DMEM with $0.8 \%$ carboxymethylcellulose, 5\% FBS, $2 \mathrm{mM}$ L-glutamine, $200 \mathrm{units} / \mathrm{ml}$ penicillin $200 \mu \mathrm{g} / \mathrm{ml}$ streptomycin) was added. Plates were left for $48 \mathrm{~h}$, overlay medium was removed and cells washed twice in PBS. Cells were fixed with methanol for $10 \mathrm{~min}$ at $4{ }^{\circ} \mathrm{C}$, washed with $150 \mu \mathrm{l}$ PBS and permeabilised for 5 min with $150 \mu \mathrm{l} 0.1 \%$ Triton X-100 (Fisher). Permeabilisation solution was removed and $35 \mu \mathrm{l}$ of 1:500 anti-DENV antibody was added (MAB8705, Millipore), diluted in $0.2 \%$ bovine serum albumin (BSA) PBS. Plates were incubated for $1.5 \mathrm{~h}$ at RT, followed by 3 washes of $100 \mu \mathrm{l}$ with $0.2 \%$ BSA PBS. Cells were incubated with $35 \mu$ of 1:400 secondary antibody (Goat anti-mouse Alexa Fluor 488, A-11001, Thermo Fisher) in 0.2\% BSA PBS for $1 \mathrm{~h}$ at RT. Cells were washed 3 times with $0.2 \%$ BSA PBS and fluorescent foci were counted using a fluorescent microscope or plates were imaged with a Typhoon FLA 9500 scanner using filter settings for Cy2. Plate images were processed in ImageJ, the 'Analyze Particles' feature was used to count fluorescent foci.

For the DENV rescue experiments, Aag2 cells were seeded in 96-well tissue culture plates (Corning) at a density of 150,000 cells $/ \mathrm{ml}$. The next day treatment commenced with 2-hydroxypropyl- $\beta$-cyclodextrin (Sigma) at the indicated concentrations in cell culture medium. After $48 \mathrm{~h}$ of treatment, cell culture medium was removed, cells were washed with $100 \mu \mathrm{PBS}$ and DENV was added in Schneider's Drosophila medium without FBS at a multiplicity of infection of 0.1 in a volume of $35 \mu \mathrm{l} /$ well. Plates were placed on an orbital shaker set at 20 r.p.m. for 2 $\mathrm{h}$ at RT. Virus was removed and normal Schneider's Drosophila medium was added with $10 \% \mathrm{FBS}$ and $200 \mathrm{units} / \mathrm{ml}$ penicillin $200 \mu \mathrm{g} / \mathrm{ml}$ streptomycin (Sigma) Plates were incubated at $28^{\circ} \mathrm{C}$ for 5 days after which cells were harvested in $100 \mu \mathrm{l}$ Tri reagent (Sigma) and RNA extracted as described below. For quantification of DENV by FFA, supernatant was harvested 7 days post infection. 
Mosquito line and rearing. A wMel-infected Ae. aegypti line was created by microinjecting wild-type embryos from a Malaysian-derived line with cytoplasm from an Indonesian-derived (UJU) $w \mathrm{Mel}$ Ae. albopictus line ${ }^{6}$. The line was outcrossed with males of the wild-type line for three generations, and maintained for $\sim 20$ generations. Wolbachia density in the $w \mathrm{Mel}$ line midguts was $7.8+-4.6$ (S. D., $n=5)$ Wolbachia surface protein/homothorax (wsp/HTH) as measured by qPCR (see below). Maternal transmission was $100 \%$ as determined by PCR. Ae. aegypti wild type and $w \mathrm{Mel}$-infected lines were maintained at $27^{\circ} \mathrm{C}$ at $70 \%$ humidity.

RNA/DNA extraction and qPCR. For estimation of Wolbachia density, DNA was extracted using phenol/chloroform from three independent samples of the Aag2 wMelPop cell line. Absolute Wolbachia density was measured by qPCR using primers targeting $w s p$ (forward: 5'-GCATCTTTTATAGCTGGTGG-3', reverse: 5'-AAAGTCCTTCAACATCAACCC-3', normalised to host $H T H$ (forward: 5'-TGGTCCTATATTGGCGGAGCTA-3', reverse: 5'-TCGTTTTTGCAAGAAGGTCA- $\left.3^{\prime}\right)^{16}$. Relative Wolbachia density in the 2 HPCD experiments was measured by qPCR using primers targeting $w s p$, normalised to host rp49 (forward: 5'-GCTATGACAAGCTTGCCCCCA-3', reverse: 5'-TCATCAGCACCTCCAGCTC- $\left.3^{\prime}\right)^{59}$

Data availability. The data from mass spectrometry are deposited at the ProteomeXchange Consortium via the PRIDE partner repository with the data set identifiers PXD003429 and PXD006239. The authors declare that all other data supporting the findings of this study are available within the article and its Supplementary Information files, or are available from the authors on request.

Received: 5 October 2016 Accepted: 11 July 2017

Published online: 13 September 2017

\section{References}

1. Hoffmann, A. A. \& Turelli, M. in Influential Passengers: Microorganisms and Invertebrate Reproduction (Oxford University Press, 1997).

2. Moreira, L. A. et al. A Wolbachia symbiont in Aedes aegypti limits infection with dengue, Chikungunya, and Plasmodium. Cell 139, 1268-1278 (2009).

3. Bian, G., Xu, Y., Lu, P., Xie, Y. \& Xi, Z. The endosymbiotic bacterium Wolbachia induces resistance to dengue virus in Aedesaegypti. PLoS Pathog. 6, e1000833 (2010)

4. Kambris, Z., Cook, P. E., Phuc, H. K. \& Sinkins, S. P. Immune activation by lifeshortening Wolbachia and reduced filarial competence in mosquitoes. Science 326, 134-136 (2009).

5. Walker, T. et al. The $w \mathrm{Mel}$ Wolbachia strain blocks dengue and invades caged Aedes aegypti populations. Nature 476, 450-453 (2011).

6. Blagrove, M. S., Arias-Goeta, C., Failloux, A. B. \& Sinkins, S. P. Wolbachia strain $w \mathrm{Mel}$ induces cytoplasmic incompatibility and blocks dengue transmission in Aedesalbopictus. Proc. Natl Acad. Sci. USA 109, 255-260 (2012).

7. Blagrove, M. S., Arias-Goeta, C., Di Genua, C., Failloux, A. B. \& Sinkins, S. P. A. Wolbachiaw Mel transinfection in Aedesalbopictus is not detrimental to host fitness and inhibits Chikungunya virus. PLoS Negl. Trop. Dis. 7, e2152 (2013).

8. van den Hurk, A. F. et al. Impact of Wolbachia on infection with chikungunya and yellow fever viruses in the mosquito vector Aedes aegypti. PLoS Negl. Trop. Dis. 6, e1892 (2012).

9. Hoffmann, A. A. et al. Successful establishment of Wolbachia in Aedes populations to suppress dengue transmission. Nature 476, 454-457 (2011).

10. Hoffmann, A. A. et al. Stability of the wMel Wolbachia infection following invasion into Aedes aegypti populations. PLoS Negl. Trop. Dis. 8, e3115 (2014).

11. Ye, Y. H. et al. Wolbachia reduces the transmission potential of dengue-infected Aedes aegypti. PLoS Negl. Trop. Dis. 9, e0003894 (2015)

12. Ferguson, N. M. et al. Modeling the impact on virus transmission of Wolbachia-mediated blocking of dengue virus infection of Aedes aegypti. Sci. Transl. Med. 7, 279ra237 (2015).

13. Teixeira, L., Ferreira, A. \& Ashburner, M. The bacterial symbiont Wolbachia induces resistance to RNA viral infections in Drosophila melanogaster. PLoS Biol. 6, e2 (2008).

14. Frentiu, F. D. et al. Limited dengue virus replication in field-collected Aedesaegypti mosquitoes infected with Wolbachia. PLoS Negl. Trop. Dis. 8 , e2688 (2014).

15. Pan, X. et al. Wolbachia induces reactive oxygen species (ROS)-dependent activation of the Toll pathway to control dengue virus in the mosquito Aedes aegypti. Proc. Natl Acad. Sci. USA 109, E23-E31 (2012).

16. Molloy, J. C. \& Sinkins, S. P. Wolbachia do not induce reactive oxygen speciesdependent immune pathway activation in Aedesalbopictus. Viruses 7 , 4624-4639 (2015)
17. Rances, E. et al. The toll and Imd pathways are not required for Wolbachiamediated dengue virus interference. J. Virol. 87, 11945-11949 (2013).

18. Lin, M. \& Rikihisa, Y. Ehrlichia chaffeensis and Anaplasma phagocytophilum lack genes for lipid A biosynthesis and incorporate cholesterol for their survival. Infect. Immun. 71, 5324-5331 (2003).

19. Wu, M. et al. Phylogenomics of the reproductive parasite Wolbachia pipientisw Mel: a streamlined genome overrun by mobile genetic elements. PLoS Biol. 2, E69 (2004).

20. Caragata, E. P. et al. Dietary cholesterol modulates pathogen blocking by Wolbachia. PLoS Pathog. 9, e1003459 (2013).

21. Heaton, N. S. et al. Dengue virus nonstructural protein 3 redistributes fatty acid synthase to sites of viral replication and increases cellular fatty acid synthesis. Proc. Natl Acad. Sci. USA 107, 17345-17350 (2010).

22. Kozik, P. et al. A human genome-wide screen for regulators of clathrin-coated vesicle formation reveals an unexpected role for the V-ATPase. Nat. Cell Biol. 15, 50-60 (2013).

23. Voronin, D., Cook, D. A., Steven, A. \& Taylor, M. J. Autophagy regulates Wolbachia populations across diverse symbiotic associations. Proc. Natl Acad. Sci. USA 109, E1638-E1646 (2012).

24. Ron, D. \& Walter, P. Signal integration in the endoplasmic reticulum unfolded protein response. Nat. Rev. Mol. Cell Biol. 8, 519-529 (2007).

25. Ferris, S. P., Jaber, N. S., Molinari, M., Arvan, P. \& Kaufman, R. J. UDP-glucose: glycoprotein glucosyltransferase (UGGT1) promotes substrate solubility in the endoplasmic reticulum. Mol. Biol. Cell 24, 2597-2608 (2013).

26. Marshall, R. D. The nature and metabolism of the carbohydrate-peptide linkages of glycoproteins. Biochem. Soc. Symp. 40, 17-26 (1974).

27. Karten, B., Peake, K. B. \& Vance, J. E. Mechanisms and consequences of impaired lipid trafficking in Niemann-Pick type C1-deficient mammalian cells. Biochim. Biophys. Acta 1791, 659-670 (2009).

28. Ikonen, E. Cellular cholesterol trafficking and compartmentalization. Nat. Rev. Mol. Cell Biol. 9, 125-138 (2008).

29. Lee, C. J., Lin, H. R., Liao, C. L. \& Lin, Y. L. Cholesterol effectively blocks entry of flavivirus. J. Virol. 82, 6470-6480 (2008).

30. Li, Q. et al. Cholesterol overloading leads to hepatic L02 cell damage through activation of the unfolded protein response. Int. J. Mol. Med. 24, 459-464 (2009).

31. Lee, J. S., Mendez, R., Heng, H. H., Yang, Z. Q. \& Zhang, K. Pharmacological ER stress promotes hepatic lipogenesis and lipid droplet formation. Am. J. Transl. Res. 4, 102-113 (2012).

32. Reyes-Del Valle, J., Chavez-Salinas, S., Medina, F. \& Del Angel, R. M. Heat shock protein 90 and heat shock protein 70 are components of dengue virus receptor complex in human cells. J. Virol. 79, 4557-4567 (2005).

33. Chatterjee, P. K., Eng, C. H. \& Kielian, M. Novel mutations that control the sphingolipid and cholesterol dependence of the Semliki Forest virus fusion protein. J. Virol. 76, 12712-12722 (2002).

34. Liu, B. Therapeutic potential of cyclodextrins in the treatment of Niemann-Pick type C disease. Clin. Lipidol. 7, 289-301 (2012).

35. Christian, A. E., Haynes, M. P., Phillips, M. C. \& Rothblat, G. H. Use of cyclodextrins for manipulating cellular cholesterol content. J. Lipid. Res. 38, 2264-2272 (1997).

36. McCauliff, L. A., Xu, Z. \& Storch, J. Sterol transfer between cyclodextrin and membranes: similar but not identical mechanism to NPC2-mediated cholesterol transfer. Biochemistry 50, 7341-7349 (2011).

37. Atger, V. M. et al. Cyclodextrins as catalysts for the removal of cholesterol from macrophage foam cells. J. Clin. Invest. 99, 773-780 (1997).

38. Liu, B. et al. Reversal of defective lysosomal transport in NPC disease ameliorates liver dysfunction and neurodegeneration in the npc1-/- mouse. Proc. Natl Acad. Sci. USA 106, 2377-2382 (2009).

39. Greenspan, P., Mayer, E. P. \& Fowler, S. D. Nile red: a selective fluorescent stain for intracellular lipid droplets. J. Cell Biol. 100, 965-973 (1985).

40. Brackney, D. E., Foy, B. D. \& Olson, K. E. The effects of midgut serine proteases on dengue virus type 2 infectivity of Aedes aegypti. Am. J. Trop. Med. Hyg. 79, 267-274 (2008)

41. Schuchman, E. H. Acid sphingomyelinase, cell membranes and human disease: lessons from Niemann-Pick disease. FEBS Lett. 584, 1895-1900 (2010).

42. Rances, E., Ye, Y. H., Woolfit, M., McGraw, E. A. \& O’Neill, S. L. The relative importance of innate immune priming in Wolbachia-mediated dengue interference. PLoS Pathog. 8, e1002548 (2012).

43. Rainey, S. M. et al. Wolbachia blocks viral genome replication early in infection without a transcriptional response by the endosymbiont or host small RNA pathways. PLoS Pathog. 12, e1005536 (2016).

44. Kolter, T. \& Sandhoff, K. Lysosomal degradation of membrane lipids. FEBS Lett. 584, 1700-1712 (2010).

45. Molloy, J. C., Sommer, U., Viant, M. R. \& Sinkins, S. P. Wolbachia modulates lipid metabolism in Aedes albopictus mosquito cells. Appl. Environ. Microbiol. 82, 3109-3120 (2016).

46. Poh, M. K. et al. U18666A, an intra-cellular cholesterol transport inhibitor, inhibits dengue virus entry and replication. Antiviral Res. 93, 191-198 (2012). 
47. Welsch, S. et al. Composition and three-dimensional architecture of the dengue virus replication and assembly sites. Cell Host Microbe 5, 365-375 (2009).

48. White, P. M. et al. Reliance of Wolbachia on high rates of host proteolysis revealed by a genome-wide RNAi screen of Drosophila cells. Genetics 205, 1473-1488 (2017).

49. Samsa, M. M. et al. Dengue virus capsid protein usurps lipid droplets for viral particle formation. PLoS Pathog. 5, e1000632 (2009).

50. Pinto, S. B., Mariconti, M., Bazzocchi, C., Bandi, C. \& Sinkins, S. P. Wolbachia surface protein induces innate immune responses in mosquito cells. BMC Microbiol. 12, S11 (2012).

51. Smyth, G. K. Linear models and empirical bayes methods for assessing differential expression in microarray experiments. Stat. Appl. Genet. Mol. Biol. 3, Article3 (2004).

52. Boersema, P. J., Raijmakers, R., Lemeer, S., Mohammed, S. \& Heck, A. J. Multiplex peptide stable isotope dimethyl labeling for quantitative proteomics. Nat. Protoc. 4, 484-494 (2009).

53. Benjamini, Y. \& Hochberg, Y. Controlling the false discovery rate: a practical and powerful approach to multiple testing. J. R. Stat. Soc. B (Methodol.) 57, 289-300 (1995)

54. Nesvizhskii, A. I., Keller, A., Kolker, E. \& Aebersold, R. A statistical model for identifying proteins by tandem mass spectrometry. Anal. Chem. 75, 4646-4658 (2003).

55. Oberg, A. L. et al. Statistical analysis of relative labeled mass spectrometry data from complex samples using ANOVA. J. Proteome Res. 7, 225-233 (2008).

56. Vizcaino, J. A. et al. ProteomeXchange provides globally coordinated proteomics data submission and dissemination. Nat. Biotechnol. 32, 223-226 (2014).

57. Sankaranarayanan, S. et al. A sensitive assay for ABCA1-mediated cholesterol efflux using BODIPY-cholesterol. J. Lipid Res. 52, 2332-2340 (2011).

58. Payne, A. F., Binduga-Gajewska, I., Kauffman, E. B. \& Kramer, L. D Quantitation of flaviviruses by fluorescent focus assay. J. Virol. Methods $\mathbf{1 3 4}$, 183-189 (2006)

59. Gentile, C., Rivas, G. B., Meireles-Filho, A. C., Lima, J. B. \& Peixoto, A. A. Circadian expression of clock genes in two mosquito disease vectors: cry2 is different. J. Biol. Rhythms 24, 444-451 (2009).

\section{Acknowledgements}

We thank H.L. Lee for providing Ae. aegypti and C. Loney for providing expertise and assistance with confocal microscopy. The study was supported by Wellcome Trust awards WT095121MA, WT202888/Z/16/Z, MRC award MC_PC_15087 and EU-FP7 award 'InfraVec'.

\section{Author contributions}

V.G. conceived experiments, performed proteomic and functional analyses; K.S. conceived experiments, carried out initial cyclodextrin and cholesterol supplementation experiments; S.M.R. performed microscopy; T.A. created the $w \mathrm{Mel} \mathrm{Ae.} \mathrm{aegypti} \mathrm{line} \mathrm{and}$ dissected midguts; A.A.D., T.L., S.H., P.D.C. and B.T. assisted with proteomic analysis; S.P.S. conceived experiments and oversaw the project; V.G. and S.P.S. wrote the manuscript and all authors checked and contributed to manuscript writing.

\section{Additional information}

Supplementary Information accompanies this paper at doi:10.1038/s41467-017-00610-8

Competing interests: The authors declare no competing financial interests.

Reprints and permission information is available online at http://npg.nature.com/ reprintsandpermissions/

Publisher's note: Springer Nature remains neutral with regard to jurisdictional claims in published maps and institutional affiliations.

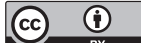

Open Access This article is licensed under a Creative Commons Attribution 4.0 International License, which permits use, sharing, adaptation, distribution and reproduction in any medium or format, as long as you give appropriate credit to the original author(s) and the source, provide a link to the Creative Commons license, and indicate if changes were made. The images or other third party material in this article are included in the article's Creative Commons license, unless indicated otherwise in a credit line to the material. If material is not included in the article's Creative Commons license and your intended use is not permitted by statutory regulation or exceeds the permitted use, you will need to obtain permission directly from the copyright holder. To view a copy of this license, visit http://creativecommons.org/ licenses/by/4.0/.

(c) The Author(s) 2017 